\title{
Los sospechosos de siempre y los inesperados Lo que el análisis de redes puede decirnos sobre la integración de políticas climáticas
}

\author{
Emilia Pramova', Bruno Locatelli,2, Monica Di Gregorio ${ }^{3,4}$, Maria Brockhaus5,6
}

\section{Mensajes clave}

- Entender los vínculos que existen entre adaptación y mitigación ayuda a identificar los cobeneficios y reduce las interacciones negativas entre estos dos ámbitos del cambio climático.

- Entre las barreras se encuentran el aislamiento institucional y la falta de información: los actores que trabajan en adaptación no están bien informados sobre las acciones de mitigación y viceversa.

- Los análisis de redes de políticas nos permiten ver con mayor claridad las interacciones que existen entre los actores que trabajan en adaptación y aquellos que trabajan en mitigación, y lo que se puede hacer para mejorarlas.

- También, nos muestran a los actores de siempre y a los inesperados que pueden facilitar los vínculos entre estos dos ámbitos.

- Este InfoBrief resume los hallazgos de un análisis de redes de políticas climáticas llevado a cabo en Perú y publicado en la revista Climate Policy (Locatelli et al. 2020).

\section{Introducción}

En una de las escenas clave de la legendaria película Casablanca (1942), el capitán Louis Renault ordena a sus oficiales de policía "rodear a los sospechosos de siempre" en lugar de arrestar a Rick Blaine, el culpable. Con frecuencia, quienes trabajamos en el tema del cambio climático hacemos lo mismo. Aunque las acciones políticas de adaptación y mitigación no están relacionadas con actividades criminales, tendemos a pensar en los mismos términos, es decir, tendemos a identificar a los "sospechosos de siempre" cuando buscamos a los campeones climáticos o a aquellos que tienen la clave para lograr el cambio; y tendemos a ignorar a los "sospechosos inesperados", aquellos que son invisibles o de los que no hemos escuchado, pero que pueden posibilitar o hacer fracasar las acciones climáticas (ver figura 1).

CIFOR, Perú

CIRAD, Universidad de Montpellier, Francia

3 Instituto de Investigación sobre Sostenibilidad, Universidad de Leeds, Reino Unido

4 CIFOR, Indonesia
¿Por qué necesitamos tanto adaptación como mitigación? La temperatura global actual es $1,3{ }^{\circ} \mathrm{C}$ más alta que la temperatura global durante la era preindustrial, y el mundo está en rumbo de alcanzar, por lo menos, $3{ }^{\circ} \mathrm{C}$ de calentamiento global (Lenton et al. 2019). La adaptación es una necesidad, así como también lo son acciones contundentes de mitigación, y estas dos estrategias esenciales para combatir al cambio climático están estrechamente relacionadas. Esto es especialmente evidente en los sectores del uso de la tierra. Por ejemplo, los sistemas agrícolas y forestales son vulnerables al cambio climático, pero también pueden ayudar a las personas a adaptarse. Al mismo tiempo, ambos sistemas son sumideros y fuentes importantes de gases de efecto invernadero.

\footnotetext{
5 Departamento de Ciencias Forestales, Universidad de Helsinki, Finlandia

6 Instituto de Helsinki de Ciencias Sostenibles (HELSUS, por sus siglas en inglés), Universidad de Helsinki, Finlandia
} 


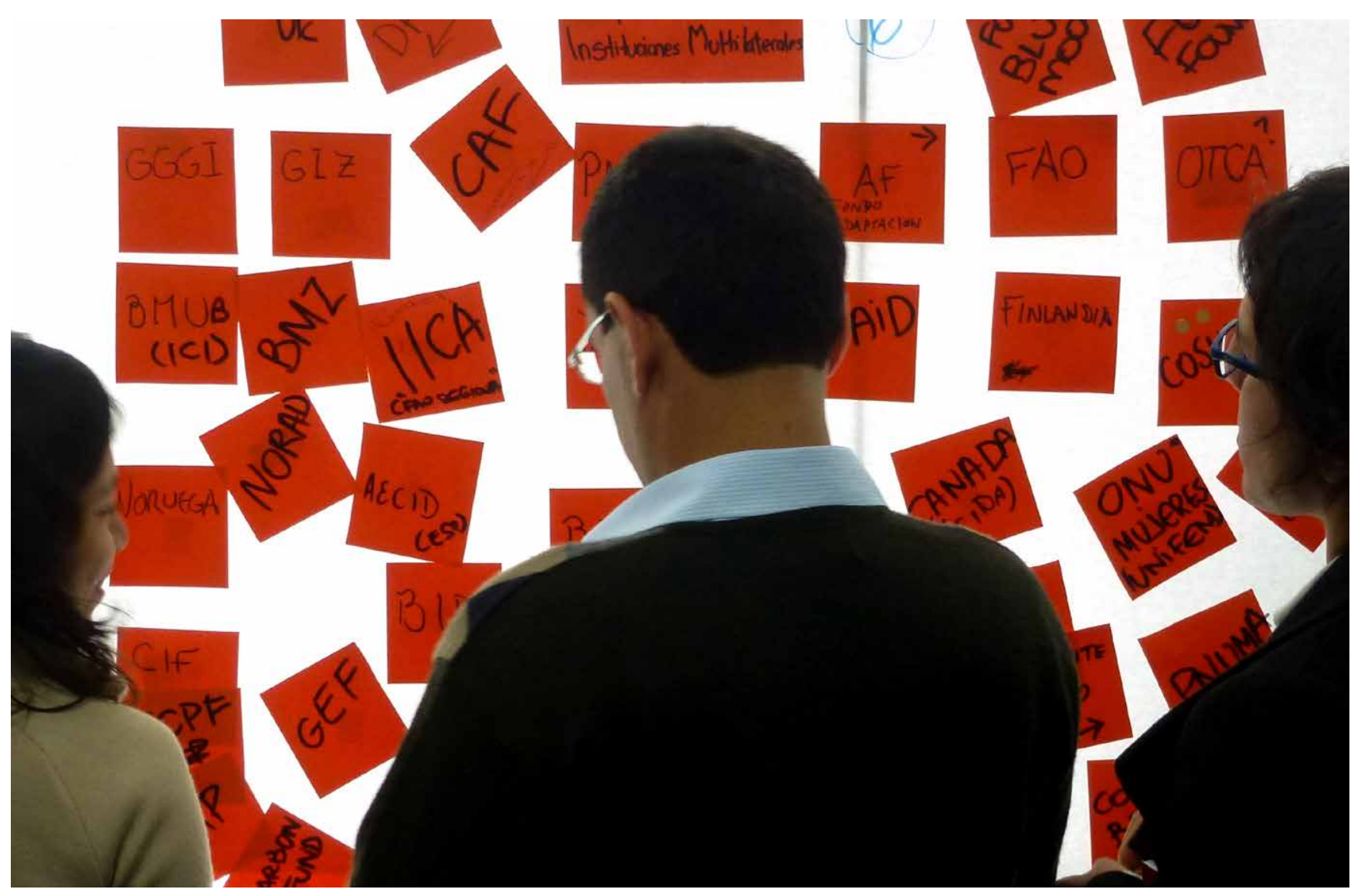

Figura 1. Durante un taller sobre redes de políticas climáticas en Lima, Perú, se pidió a los participantes identificar a los sospechosos usuales y a los inesperados. (Foto por Bruno Locatelli).

Una acción climática particularmente relacionada con el uso de la tierra puede producir cobeneficios para la adaptación, la mitigación y el desarrollo sostenible, pero también puede traer consecuencias no deseadas (Locatelli et al. 2015). La integración de los objetivos y los procesos de las políticas de adaptación y mitigación climática ayuda a identificar estas prácticas mutuamente beneficiosas y reduce las interacciones negativas. Esto significa incluir objetivos conjuntos de adaptación y mitigación en el diseño de intervenciones, en donde sea pertinente, y asegurar que los actores relacionados con las políticas interesados ya sea en adaptación o mitigación coordinen entre ellos (Di Gregorio et al. 2017). Además, se necesita integrar al cambio climático en los procesos de políticas de otros sectores.

El primer paso consistiría en facilitar la comunicación y la coordinación entre los actores de políticas que participan en procesos de políticas de adaptación y mitigación climáticas. Esto, sin embargo, es muy difícil de lograr. Usualmente, las políticas nacionales y los programas se enfocan en solo una estrategia, ya sea adaptación o mitigación, y quienes toman las decisiones tienden a trabajar solo al interior de sus propios sectores. Con frecuencia, las personas que se involucran en el tema de la adaptación no están completamente informadas sobre los procesos de mitigación y viceversa.

Las barreras institucionales que separan a los ámbitos de la adaptación y la mitigación, las diferencias en el conocimiento sobre las necesidades de adaptación y mitigación, y la escasa capacidad para la coordinación son los desafíos que caracterizan a los procesos de políticas relacionados con el clima. Los análisis de redes de políticas pueden revelar algunas de las barreras, y ofrecer algunas ideas sobre posibles oportunidades para mejorar la comunicación y la coordinación entre los actores. Asimismo, pueden dejar en claro quiénes son los actores están en una mejor posición para conectar los ámbitos de las políticas de adaptación y mitigación (tanto a los sospechosos de siempre, como a los inesperados).

Este brief resume los resultados de un análisis de redes de políticas realizado en Perú y publicado en la revista Climate Policy (Locatelli et al. 2020). 


\section{¿Por qué un análisis de redes de políticas?}

Las redes de políticas climáticas involucran a actores estatales y no estatales de diferentes sectores y niveles, y cada uno de ellos busca influir en los procesos en curso relacionados con políticas y sus resultados. Los análisis de redes de políticas tienen su origen en los análisis de redes sociales. Investigan cómo los actores relevantes interactúan, con el objetivo de revelar patrones sociales, las interdependencias de los recursos y las relaciones de poder en la elaboración y la implementación de políticas de cambio climático (Brockhaus et al. 2014). También, nos dan a conocer quiénes son los actores dominantes, quiénes son los más aislados, y quiénes son los mediadores que pueden promover nuevas colaboraciones o relaciones entre los diferentes grupos (también conocidos como brókeres), y las estructuras de las redes que las facilitan o las limitan.

Esta información puede ser valiosa para entender las fallas en el funcionamiento y para diseñar estrategias que puedan mejorar las redes institucionales. Por ejemplo, en el caso de actores aislados se pueden concebir estrategias para construir relaciones interorganizacionales, ya que son un aspecto importante de la gobernanza climática. La complejidad de la gobernanza climática exige negociar con los diversos intereses y resolver conflictos entre los diferentes sectores y actores relacionados con las políticas, desde el nivel local hasta el internacional. Trabajar para lograr la integración de políticas climáticas requiere: 1) entender si los procesos de políticas de adaptación y mitigación están o no conectados, y si están conectados, descubrir de qué forma; y 2) entender cómo está estructurado el ámbito de políticas de cambio climático.

\section{Interacciones entre la adaptación y la mitigación en las redes de políticas}

\begin{abstract}
En Perú, para documentar las interacciones entre los actores involucrados en la adaptación y en la mitigación, hicimos una encuesta y realizamos entrevistas semiestructuradas con representantes de 76 organizaciones que participan en procesos de políticas a nivel nacional. Pedimos a los representantes señalar a los actores con los que, de manera regular, su organización intercambia información sobre adaptación y a aquellos con los que ha colaborado. Hicimos lo mismo en lo que se refiere a mitigación, y además preguntamos a cuáles organizaciones percibían como particularmente influyentes en procesos de políticas de adaptación, y a cuáles en los procesos de mitigación.
\end{abstract}

\begin{abstract}
De este modo, obtuvimos seis diferentes relaciones para nuestro análisis: intercambio de información en 1) adaptación, 2) mitigación; colaboración en cuestiones de 3) adaptación o 4) mitigación; e influencia percibida en el ámbito de la 5) adaptación o 6) mitigación. Representamos a cada ámbito, el de la adaptación y el de la mitigación, como una "capa" diferente en la red de políticas climáticas, la que construimos como una "red de múltiples capas" o "multilayer network" en inglés (Dickison 2016). Cada capa la exploramos de forma separada y de forma integrada; y examinamos las dinámicas entre las capas para encontrar a nuestros sospechosos, es decir, cuáles organizaciones promovieron relaciones al interior y entre los dos ámbitos, el de la adaptación y el de la mitigación.
\end{abstract}

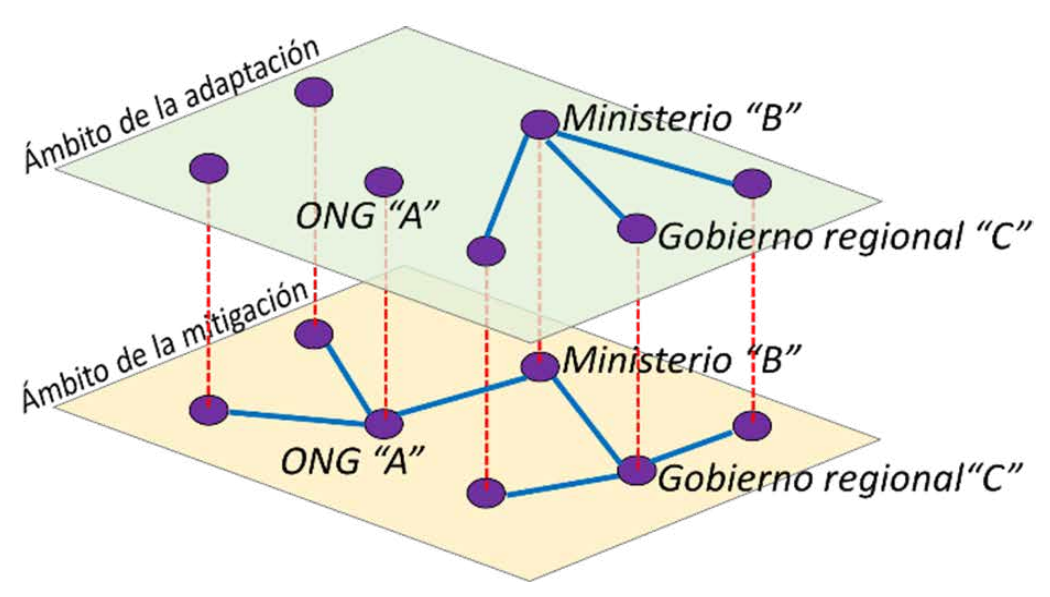

Figura 2. Red estilizada de múltiples capas. Los mismos actores están en cada capa. Las líneas azules muestran sus interacciones con otros actores. Cada línea vertical, en rojo, conecta al mismo actor en las dos capas de los ámbitos de adaptación y mitigación. 
En una red de múltiples capas, los actores de políticas tienen más que una relación; por ejemplo, una organización puede estar conectada a otra por los flujos de comunicación, por proyectos conjuntos o por ambos. En nuestro caso, los actores de políticas están conectados por intercambio de información y colaboración relacionada con la adaptación y/o la mitigación. En un ejemplo ficticio y sencillo (figura 2), los actores están representados con puntos morados y están conectados con líneas azules (lazos de información o colaboración) en dos capas: adaptación y mitigación.

Los mismos actores están presentes en las dos capas, adaptación y mitigación), dado que todos están involucrados en procesos de políticas sobre cambio climático. Algunos de ellos podrían estar inactivos en alguno de las dos capas. Por ejemplo, la organización no gubernamental (ONG) "A" no interactúa con ninguna entidad en temas de adaptación. El ministerio "B" es un actor central en adaptación y también es un bróker importante que conecta a muchos actores que de otra forma podrían permanecer desconectados en esta capa. El ministerio "B" es también un bróker en la capa de mitigación y entre las dos capas. Mientras que la ONG "A", el ministerio " $B$ " y el gobierno regional " $C$ " son brókers en mitigación, la figura 2 muestra que desempeñan diferentes roles al conectar a los actores entre capas: " $A$ " no desempeña ningún papel, en tanto que " $B$ " y " $C$ " si lo hacen, aun cuando " $C$ " no es un bróker en temas de adaptación.

\section{Actores y sus conexiones}

Al comparar las redes de políticas de cambio climático de Perú, notamos que hubo más conexiones entre los actores de la capa de mitigación (mayor densidad), que entre los actores de la capa de adaptación. Ello muestra que en el momento en el que se realizaron las entrevistas había más actividad en el ámbito de la mitigación. El resultado fue el esperado, dado que, históricamente, la mitigación ha atraído mayor atención internacional y recursos que la adaptación. Además, en nuestra red, si un actor mostraba un alto nivel de poder relacionado con su reputación en la capa de mitigación, la probabilidad de que otros actores establecieran lazos con ese actor en ambas capas se incrementaba (y no solo en la de mitigación).

Del mismo modo, otro estudio (de solo unos cuantos) que analiza las interacciones entre los procesos de políticas de adaptación y mitigación demostró mayor actividad en el ámbito correspondiente a la mitigación en Brasil e Indonesia (Di Gregorio et al. 2019).

Los sospechosos de siempre en los procesos nacionales de políticas climáticas -organizaciones de gobiernos nacionales, agencias extranjeras y organizaciones intergubernamentalesfueron actores centrales en las capas de adaptación y mitigación de nuestro caso de estudio. En nuestro estudio, cuando hablamos de un "actor central", queremos decir que

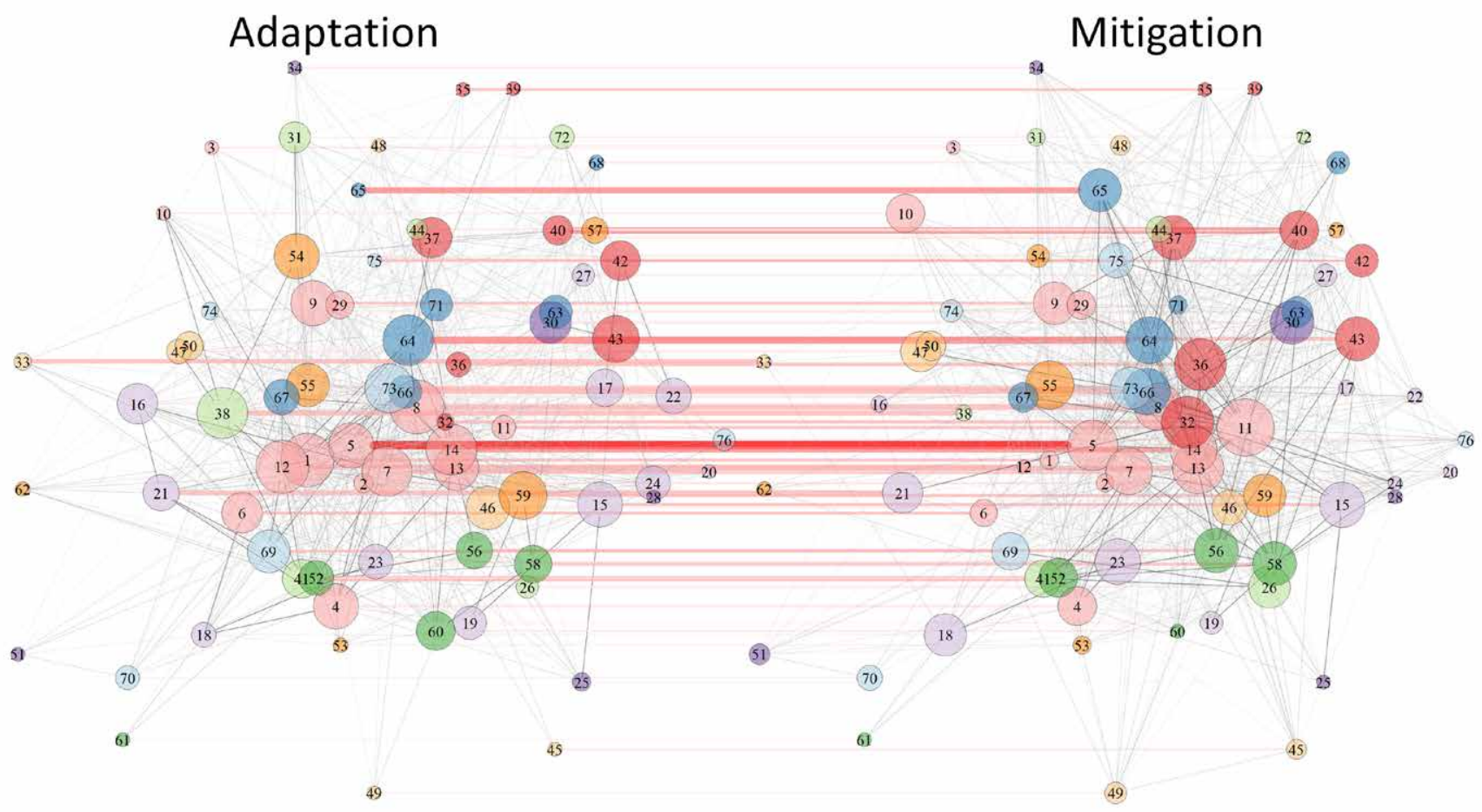

Figura 3. Representación de la red de políticas climáticas en Perú. Muestra las dos capas intercambiando información y colaborando en adaptación (izquierda) y mitigación (derecha), y los lazos que las unen. Los círculos representan actores. Los colores de los círculos representan diferentes tipos de organizaciones, por ejemplo, gobierno nacional u ONG internacionales (ver Locatelli et al. 2020, para mayores detalles). 
muchos otros actores han indicado relaciones con éste en particular (en la figura 3, el tamaño del círculo corresponde con la cantidad de relaciones). Como grupo, las organizaciones gubernamentales nacionales fueron los principales actores centrales. Esto nos da una indicación del nivel de apropiación nacional de la agenda de cambio climático en Perú, así como del buen nivel de institucionalización de respuestas al cambio climático.

En ambas capas, los actores con el menor número de conexiones fueron las grandes compañías del sector privado y las organizaciones de la sociedad civil (OSC) que operan a nivel subnacional, lo que indica el aislamiento de grupos que son importantes para la implementación de medidas de adaptación y mitigación. Sin embargo, no todos los actores que están aislados tienen la misma función y los mismos recursos, y en lo que se refiere a su poder, existen grandes asimetrías entre ellos.

Podría parecer que las grandes empresas del sector privado están aisladas ya sea porque actualmente no priorizan los temas de cambio climático (y, por lo tanto, no quieren involucrarse en ellos); porque, en los hechos, interactúan con actores centrales, aunque de forma no visible; o porque no necesitan llevar a cabo acciones (ya que sus intereses son tomados en cuenta debido a su poder y acceso a personajes políticos). Por el contrario, el aislamiento de las OSC de menor tamaño refleja la típica distribución de poder en la sociedad. Esto es problemático porque el involucramiento con las OSC, especialmente aquellas locales, es muy importante para asegurar que las medidas de cambio climático sean social y ambientalmente justas.

Encontramos que muchas organizaciones de diferentes grupos estaban bien posicionadas en la red por promover relaciones entre las capas de adaptación y mitigación. A los sospechosos de siempre los identificamos: actores con mandatos que abarcan ambos ámbitos, que son centrales y que promueven relaciones en ambas capas. Efectivamente, muchos de los principales brókeres entre las dos capas fueron actores pertenecientes al gobierno nacional y organizaciones internacionales que tenían una buena posición como intermediarios tanto en adaptación como en mitigación, o en al menos uno de los dos ámbitos. Pero también hubo algunos sospechosos inesperados que se encontraron en una buena posición para fungir como intermediarios.

Por ejemplo, entre los sospechosos inesperados encontramos a una organización de gobierno regional y a una asociación de productores, que no fueron brókeres en ninguna de las dos capas de forma separada, y tampoco fueron centrales en una o ambas de las capas. Pero, a pesar de su bajo perfil en los ámbitos de la adaptación y la mitigación, aparecieron como mediadores importantes entre la adaptación y la mitigación porque fueron el único lazo potencial entre capas y entre varios actores que de otra forma habrían permanecido desconectados.
Sorprendentemente, y contrario a esto, algunos actores centrales en ambas capas, y con un mandato de trabajar con múltiples actores y de conectarlos (por ejemplo, una agencia extranjera con un extenso portafolio de actividades climáticas en Perú), no estuvieron en las categorías más altas de brókeres entre capas. Por lo tanto, no confirmamos lo que pensábamos sobre varios de los sospechosos de siempre. Nuestros sospechosos de siempre podrían haber obtenido una calificación baja como brókeres, como resultado de su éxito: establecieron lazos y lograron que otros actores crearan lazos directos, por lo que su función como intermediarios ya no era necesaria (Burt 2009).

\section{Identificando a todos los sospechosos}

El análisis de redes de políticas es un enfoque relevante para evaluar la integración de procesos de políticas. Este enfoque puede ayudar a identificar a todos los actores que pueden promover vínculos entre los ámbitos, así como a identificar las estructuras de poder que pueden apoyar u obstaculizar una implementación más efectiva y justa de políticas y medidas climáticas. Por ejemplo, con frecuencia es necesario apoyar a los actores que tienen un papel clave en conectar a la adaptación con la mitigación, para sostener y fortalecer en mayor medida los vínculos entre los ámbitos. Esto podría significar ofrecer a algunos de los sospechosos inesperados un mejor acceso a órganos o procesos formales de cambio climático. También podría significar hacer más visibles las actividades de algunos actores, para que el papel que desempeñan y las contribuciones que realizan se entiendan mejor, y para que con ello puedan rendir cuentas de sus acciones ante aquellos con menor poder.

Los estudios de redes de políticas tales como el nuestro, ofrecen un vistazo rápido a lo que está sucediendo en cuanto a políticas de cambio climático en un momento en particular. Pero si se hace periódicamente, pueden ayudarnos a entender cómo las estructuras de redes y de poder cambian en la gobernanza climática y si la integración de políticas climáticas mejora a lo largo del tiempo.

\section{Recomendaciones}

- Por ser actores centrales, organizaciones de gobiernos nacionales pueden facilitar la comunicación y la coordinación entre otros actores involucrados en políticas y procesos de cambio climático.

- Las organizaciones de gobiernos nacionales pueden activar estructuras institucionales $u$ organismos de coordinación que incluyan y apoyen a los actores aislados (por ejemplo, organizaciones subnacionales) o a los actores que pueden llegar a muchos más (por ejemplo, los brókeres). 
- En primer lugar, estos organismos deberían promover que los actores compartan información sobre actividades y conocimientos, evitando que haya fatiga en la coordinación y sin causar altos costos de transacción.

- Los actores que están bien posicionados para promover relaciones entre organizaciones necesitarán incentivos y apoyo para involucrarse en ello.

\section{Referencias}

Brockhaus, M., Di Gregorio, M. y Carmenta, R. 2014. REDD+ policy networks: exploring actors and power structures in an emerging policy domain. Ecology and Society 19(4). https:// www.jstor.org/stable/26269649

Burt, R.S. 2009. Structural holes: the social structure of competition. Harvard: Harvard University Press.

Dickison, M.E., Magnani, M. y Rossi, L. 2016. Multilayer social networks. Cambridge: Cambridge University Press._doi. org/10.1017/CBO9781139941907

Di Gregorio, M., Nurrochmat, D.R., Paavola, J., Sari, I.M., Fatorelli, L., Pramova, E., Locatelli, B., Brockhaus, M. y Kusumadewi, S.D. 2017. Climate policy integration in the land-use sector: mitigation, adaptation and sustainable development linkages. Environmental Science \& Policy 67: 35-43. https://doi. org/10.1016/j.envsci.2016.11.004

Di Gregorio, M., Fatorelli, L., Paavola, J., Locatelli, B., Pramova, E., Nurrochmat, D.R., May, P.H., Brockhaus, M., Sari, I.M. y Kusumadewi, S.D. 2019. Multi-level governance and power in climate change policy networks. Global Environmental Change 54: 64-77. doi.org/10.1016/j.gloenvcha.2018.10.003

Lenton, T.M., Rockström, J., Gaffney, O., Rahmstorf, S., Richardson, K., Steffen, W. y Schellnhuber, H.J. 2019. Climate tipping points-too risky to bet against. Nature 575: 592-593. doi. org/10.1038/d41586-019-03595-0

Locatelli, B., Pavageau, C., Pramova, E., Di Gregorio, M., 2015. Integrating climate change mitigation and adaptation in agriculture and forestry: opportunities and trade-offs. WIRES Climate Change 6(6): 585-598. https://doi.org/10.1002/wcc.357 Locatelli, B., Pramova, E., Di Gregorio, M., Brockhaus, M., Chávez, D. A., Tubbeh, R., Sotés, J. y Perla, J. 2020. Climate change policy networks: connecting adaptation and mitigation in multiplex networks in Peru. Climate Policy: 1-19. https://doi.org /10.1080/14693062.2020.1730153

\section{Agradecimientos}

Entre los socios financieros que han apoyado esta investigación se encuentran la Iniciativa Internacional para el Clima (IKI, proyecto 15_III_075) del Ministerio Federal de Medio Ambiente, Conservación de la Naturaleza, Construcción y Seguridad Nuclear de Alemania (BMU); la Agencia Noruega de Cooperación para el Desarrollo (NORAD, acuerdo QZA-016/0110); la Agencia Nacional de Investigación de Francia (ANR, proyecto TRASSE ANR-CONACYT-17-CE32-0012); el Consejo de Investigación Económica y Social (ESRC, por sus siglas en inglés, número de financiamiento ES/K00879X/1); el Centro sobre Economía y Políticas de Cambio Climático (CCCEP, por sus siglas en inglés, número de financiamiento ES/K006576/1 del ESRC); y el Programa de Investigación del CGIAR sobre Bosques, Árboles y Agroforestería (CRP-FTA) con el apoyo financiero de los contribuyentes al Fondo Fiduciario del CGIAR. Los autores agradecen a los entrevistados por participar en este estudio.

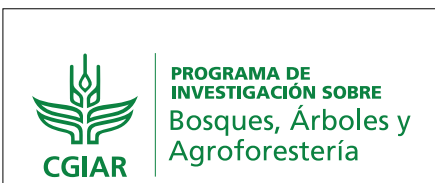

Esta investigación fue realizada por CIFOR como parte del Programa de Investigación de CGIAR sobre Bosques, Árboles y Agroforestería (FTA). El FTA es el programa de investigación para el desarrollo más grande del mundo, dedicado a mejorar el papel de bosques, árboles y la agroforestería para el desarrollo sostenible, la seguridad alimentaria, y frente al cambio climático. CIFOR dirige el programa FTA en asociación con Bioversity International, CATIE, CIRAD, ICRAF, INBAR y TBI. La investigación del Programa FTA cuenta con el apoyo del Fondo Fiduciario del CGIAR: cgiar.org/funders 